\title{
Intra-operative hyperthermic intraperitoneal chemotherapy for prevention and treatment of peritoneal metastases from gastric cancer: a narrative review
}

\author{
Zhong-He Ji ${ }^{1}$, Ying Zhang ${ }^{2}$, Yan $\mathrm{Li}^{1,2}$ \\ ${ }^{1}$ Department of Peritoneal Cancer Surgery, Cancer Center of Beijing Shijitan Hospital, Capital Medical University, Beijing, China; ${ }^{2}$ Department of \\ Pathology, Cancer Center of Beijing Shijitan Hospital, Capital Medical University, Beijing, China \\ Contributions: (I) Conception and design: Y Li, ZH Ji; (II) Administrative support: Y Li; (III) Provision of study materials or patients: ZH Ji, Y Zhang; \\ (IV) Collection and assembly of data: All authors; (V) Data analysis and interpretation: All authors; (VI) Manuscript writing: All authors; (VII) Final \\ approval of manuscript: All authors. \\ Correspondence to: Yan Li, MD, PhD. Department of Peritoneal Cancer Surgery and Pathology, Beijing Shijitan Hospital, Capital Medical University, \\ No. 10 Tieyi Road, Yangfangdian Street, Haidian District, Beijing 100038, China. Email: liyansd2@163.com.
}

\begin{abstract}
Peritoneal metastasis (PM) from gastric cancer (GC) has long been regarded as the terminal disease, lacking of effective treatments. In recent 40 years, cytoreductive surgery (CRS) plus perioperative intraperitoneal chemotherapy, including hyperthermic intraperitoneal chemotherapy (HIPEC), neoadjuvant intraperitoneal and systemic chemotherapy (NIPS), and early post-operative intraperitoneal chemotherapy (EPIC), has been recommended as a preferred treatment option for some selected patients with GCPM. Intraperitoneal free cancer cells were recognized as the pathological cause of PM and the primary target for intraperitoneal chemotherapy. There were a lot of evidence demonstrating that HIPEC could effectively eradiate intraperitoneal free cancer cells and prolong overall survival in GCPM. However, there are still no standard HIPEC protocols. This review summarized the current HIPEC regimens used in GCPM from a literature search, trying to conclude the optimal HIPEC in GCPM, and indicate the future direction of HIPEC study. Moreover, the new data on the exploration of HIPEC in GCPM at Shijitan Hospital, Capital Medical University was shared. In conclusion, there was not enough evidence from publications and our own experience to conclude a recommended HIPEC regimen for GCPM. There is urgent need for standardizing HIPEC protocols worldwide. Accordingly, more international collaborations focusing on pharmacology and HIPEC-related parameters to generate high level evidence are essential.
\end{abstract}

Keywords: Cytoreductive surgery (CRS); hyperthermic intraperitoneal chemotherapy; gastric cancer (GC); peritoneal metastasis $(\mathrm{PM})$

Submitted Jul 06, 2020. Accepted for publication Aug 25, 2020.

doi: 10.21037/jgo-20-262

View this article at: http://dx.doi.org/10.21037/jgo-20-262

\section{Introduction}

Peritoneal metastasis $(\mathrm{PM})$ is the most common, aggressive and lethal type of metastasis of gastric cancer (GC), in the era of standard D2 gastrectomy plus systemic chemotherapy, and has long been regarded as an incurable condition with rapidly deteriorating symptoms of refractory ascites, progressive intestinal obstruction, and uncontrollable abdominal pain (1).
Over the past four decades, significant improvements have been achieved in the field of peritoneal surface oncology. It is now generally accepted in the oncology community that in some highly selected patients peritoneal metastasis is a regional and treatable disease instead of a terminal disease (2). An integrated therapeutic package combining cytoreductive surgery (CRS) and perioperative intraperitoneal chemotherapy, including hyperthermic 
intraperitoneal chemotherapy (HIPEC) has been developed to treat peritoneal surface malignancies, with curative or palliative intents (3).

Recently, the Chicago Consensus on the management of GCPM recommended CRS with gastrectomy and intraperitoneal chemotherapy as the treatment for synchronous GCPM with low peritoneal cancer index following by standard chemotherapy (4). However, the drug regimen, dose, temperature, duration and other parameters of HIPEC are still not standardized.

As an established center for peritoneal surface cancer in China, with the capacity of over 200 CRS + HIPEC procedures per year, our center has long been devoted to the development, application, promotion, and education on the integrated treatment of PM from gastrointestinal and gynecological malignancies. In this review, our goal is to update our exploration of optimal HIPEC in GCPM and summarize the current HIPEC strategies from a review of the literature, trying to identify the future direction of HIPEC study in GCPM. We present the following article in accordance with the Narrative Reporting Checklist (available at http://dx. doi. org/10.21037/jgo-20-262).

\section{Rationale for HIPEC in GCPM}

Intraperitoneal free cancer cells (IFCCs) originating from spontaneous exfoliation of the primary tumour or iatrogenic dissemination at the time of surgery are the pathological cause of GCPM (5). It has been described by Yonemura et al. that IFCCs could invade into peritoneum through transmesothelial and translymphatic pathways (6) (Figure 1). In other words, IFCCs and the resulting invisible micrometastasis on peritoneal surfaces are the primary target for preventing and treating GCPM.

The presence of plasma-peritoneal barrier makes the peritoneal cavity a relatively closed space lacking blood vessels and accounting for the poor effect of intravenous chemotherapy. HIPEC is designed to exploit the function of plasma-peritoneal barrier; that is to achieve a high intraperitoneal concentration of chemotherapeutics with a low plasma concentration. This positive gradient of chemotherapy in the peritoneum will intensify its direct antitumor effect with less systemic adverse effects. Hyperthermia itself has direct detrimental effects on IFCCs and also enhances the effects of intraperitoneal chemotherapy by increasing the depth of drug penetration and the drug uptake by tumor cells (5). We reported that HIPEC will effectively eradicate IFCCs in $78 \%$ patients with peritoneal metastasis. This was determined by detecting pre- and post-HIPEC viability of IFCCs using traditional cytological assessment. However, HIPEC failed to significantly reduce the molecular markers, like CEA mRNA and CK20 mRNA (Figure 2) (7).

\section{Role of HIPEC in GCPM}

It has been accepted that CRS+HIPEC is a promising integrated treatment strategy for GCPM (1). There is no doubt that CRS plays a fundamental role in this comprehensive package, and the completeness of CRS is the dominant independent prognostic factor for overall survival (OS). However, HIPEC is also an essential complement, rather than an alternative technique in the treatment of GCPM. Table 1 lists the published controlled studies comparing CRS+HIPEC versus CRS alone in GCPM (8-15), including only 1 prospective randomized control trial (RCT) and 7 retrospective analyses. Within these studies, 6 (75\%) demonstrated that HIPEC could significantly prolong the OS for GCPM as compared to CRS alone. However, more well designed RCTs are urgently needed for verifying the role of HIPEC in the management of GCPM, due to the lower evidence level of the available trials. Currently, the GASTRIPEC trial (NCT02158988) is the only active multicenter RCT focusing on CRS+HIPEC in GCPM, aiming to compare the efficacy and safety of CRS with or without HIPEC using mitomycin $\mathrm{C}$ and cisplatin. We hope this trial may bring some new evidence into this uncertain filed.

\section{Current HIPEC regimens in GCPM from a literature search}

The optimal methodology for HIPEC remains an unsolved problem. The multiple HIPEC regimens from 29 publications are listed in Table 2. We could conclude the following characteristics. First, $65 \%(17 / 26)$ centers preferred an open technique instead of closed technique, although there was no evidence to distinguish these two methods. Moreover, the open-technique was more frequently used in Japan and China, while the closed technique was more common in USA. Second, a combined drug regimen was used more frequently than monochemotherapy. Mitomycin $\mathrm{C}$ in early years or more recently oxaliplatin nowadays has been used as a single 


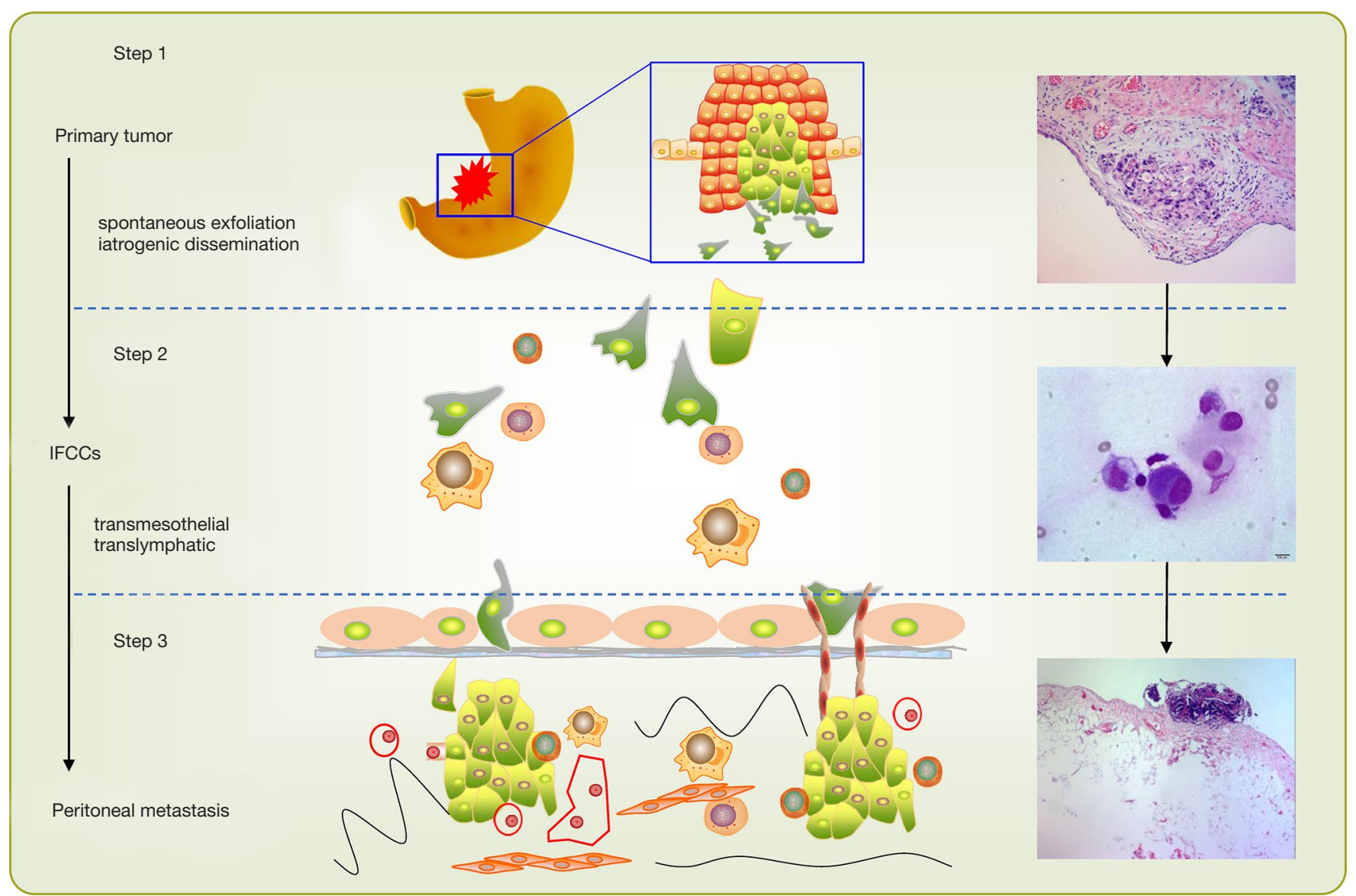

Figure 1 Mechanism of peritoneal metastasis from gastric cancer. Step 1: Cancer cell exfoliated from primary tumor, as a result of cancer invasion through serosa and/or iatrogenic dissemination during surgery. Step 2: Intraperitoneal free cancer cells (IFCCs) invade into subperitoneal space by directly interaction with mesothelium cells or through lymphatic orifices opening on peritoneal surface. Step 3: The integrity of the peritoneal-blood barrier is broken forming a favorable site for tumor proliferation. IFCCs colonize near the subperitoneal capillary, and proliferate via autocrine or paracrine stimulation by production of growth factors and neovascularization induced by angiogenic factors. The parallel pathologic image stained by HE method is shown on the right side. This figure was adapted from the original drawing of Yutaka Yonemura (6).

agent for HIPEC. The most common drug combination was cisplatin + mitomycin $\mathrm{C} \pm$ etoposide and cisplatin + doxorubicin. Third, the dose of monochemotherapy with mitomycin $\mathrm{C}$ or oxaliplatin was relatively fixed. However, the dose of combined drugs varied widely. Fourth, there was no standard temperature and duration for HIPEC. The temperature of HIPEC in 29 publications ranged from 40 to $45^{\circ} \mathrm{C}$, and the duration ranged from 30 to 120 minutes. Fifth, the flow rate, carrier solution, and other parameters were not precisely discussed in these publications. Sixth, the HIPEC regimens were relatively conservative in Asia and USA, but quite aggressive in Europe. The multicenter studies in European countries showed that the HIPEC regimens varied widely from different centers even within the same country $(8,39,40)$.

\section{Exploration of HIPEC in GCPM at Shijitan Hospital}

In 2011, our group published the first phase III RCT comparing CRS+HIPEC or CRS alone in GCPM. In this protocol we used the open-technique for HIPEC, with $120 \mathrm{mg}$ of cisplatin and $30 \mathrm{mg}$ of mitomycin $\mathrm{C}$ at $43.0 \pm 0.5^{\circ} \mathrm{C}$ for 60-90 minutes (10). The trial showed that such HIPEC regimens could significantly improve survival with acceptable morbidity in synchronous GCPM patients. 
A

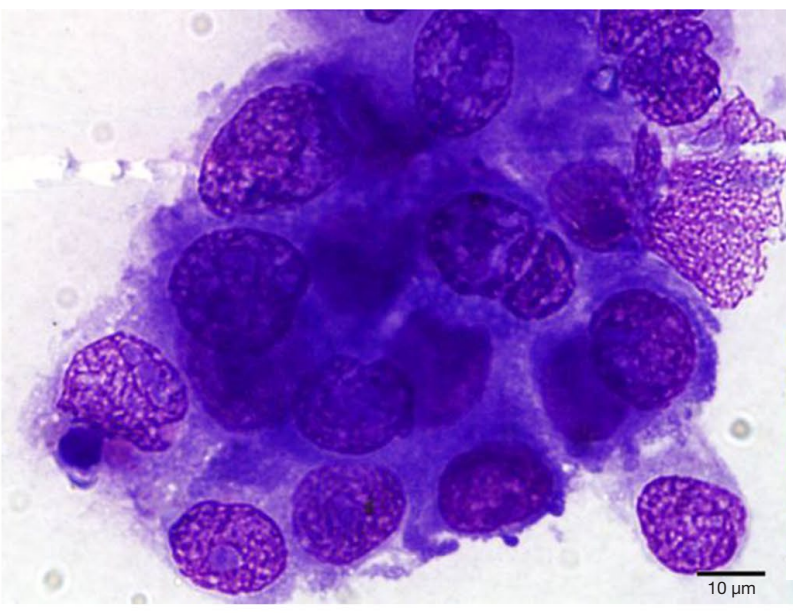

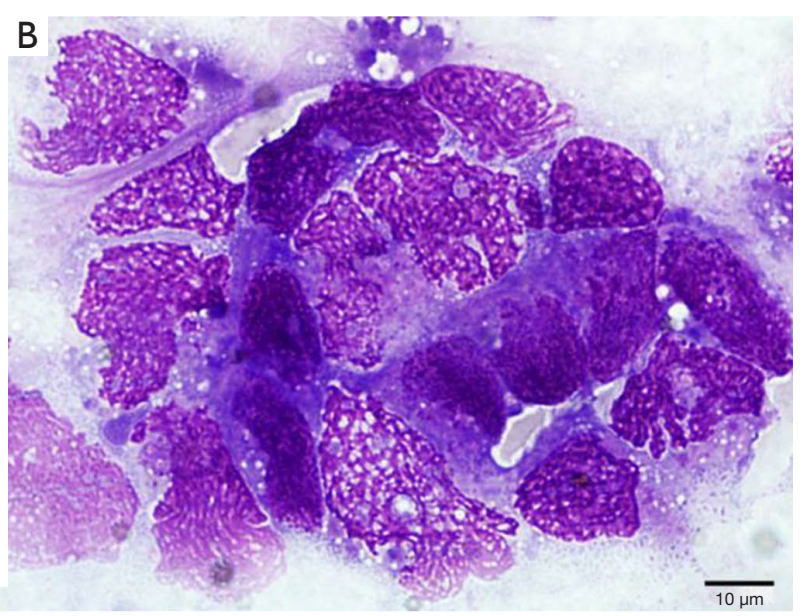

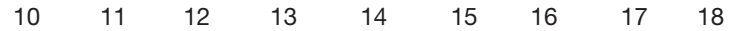

C
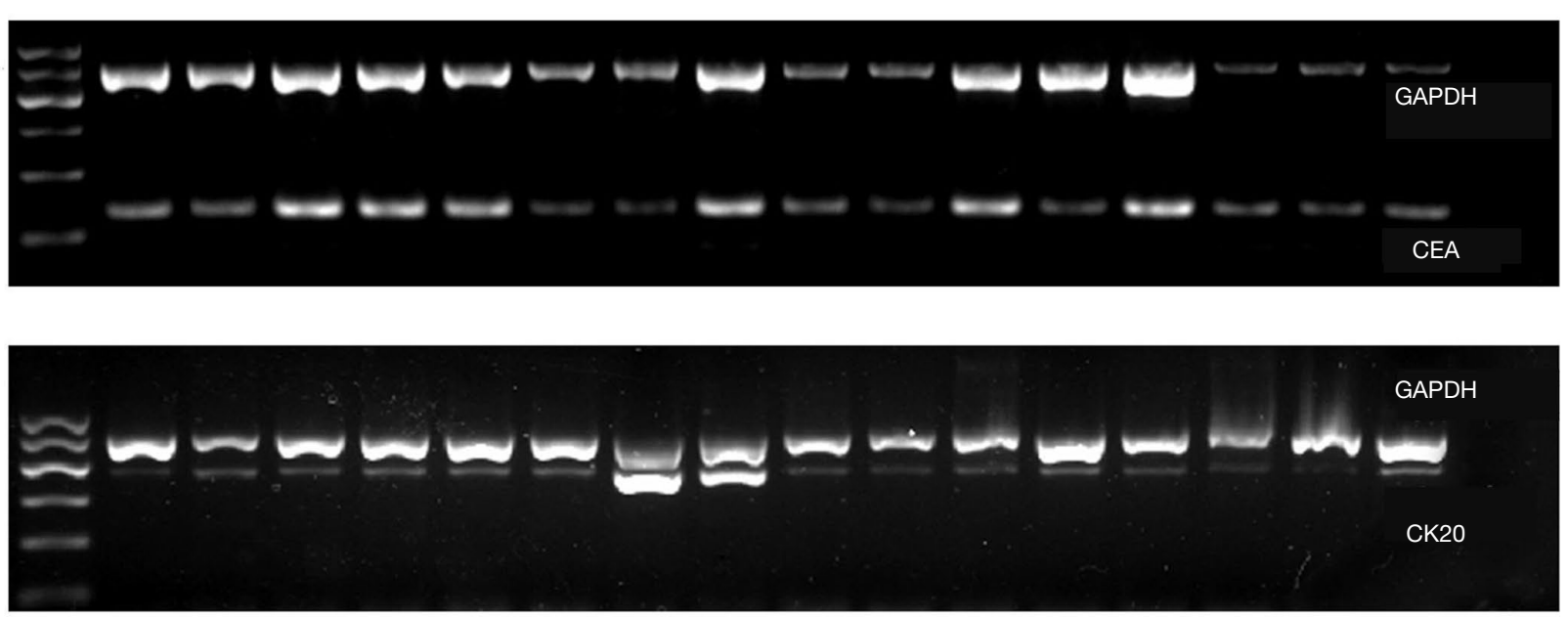

$\mathrm{D}$

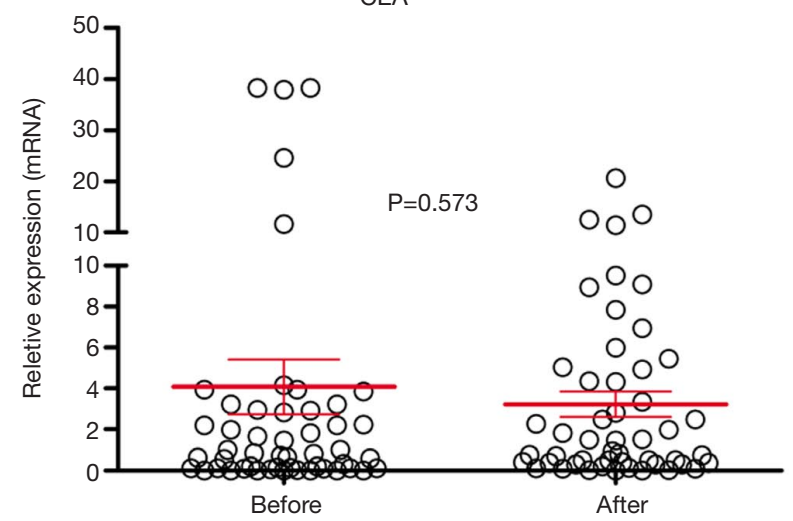

$E$

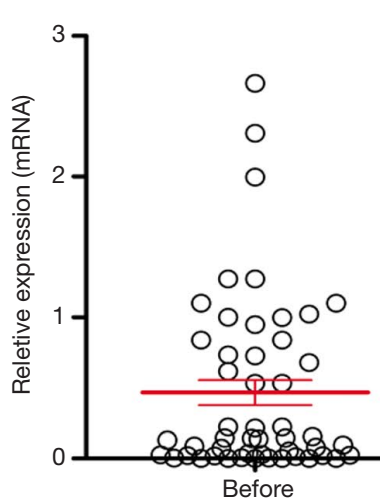

CK20

Figure 2 Assessment of hyperthermic intraperitoneal chemotherapy (HIPEC) in treating intraperitoneal free cancer cells (IFCCs) by conventional cytology (A: IFCCs before HIPEC, B: IFCCs after HIPEC) and RT-PCR method (C: Agarose gel electrophoresis of the product of CEA and CK20, D: CEAmRNA value before and after HIPEC, E: CK20 mRNA value before and after HIPEC). wright's stain, $1,000 \times$, scale bar $=10 \mu \mathrm{m}$ in A and B. Lane 1 is a DNA maker from $100 \mathrm{bp}$ (bottom) to $600 \mathrm{bp}$ (top); lanes 3 to 17 are the patients' samples; lane 2 is the positive control; lane 18 is the negative control in C. Horizontal lines represent mean \pm standard error in D and E. 


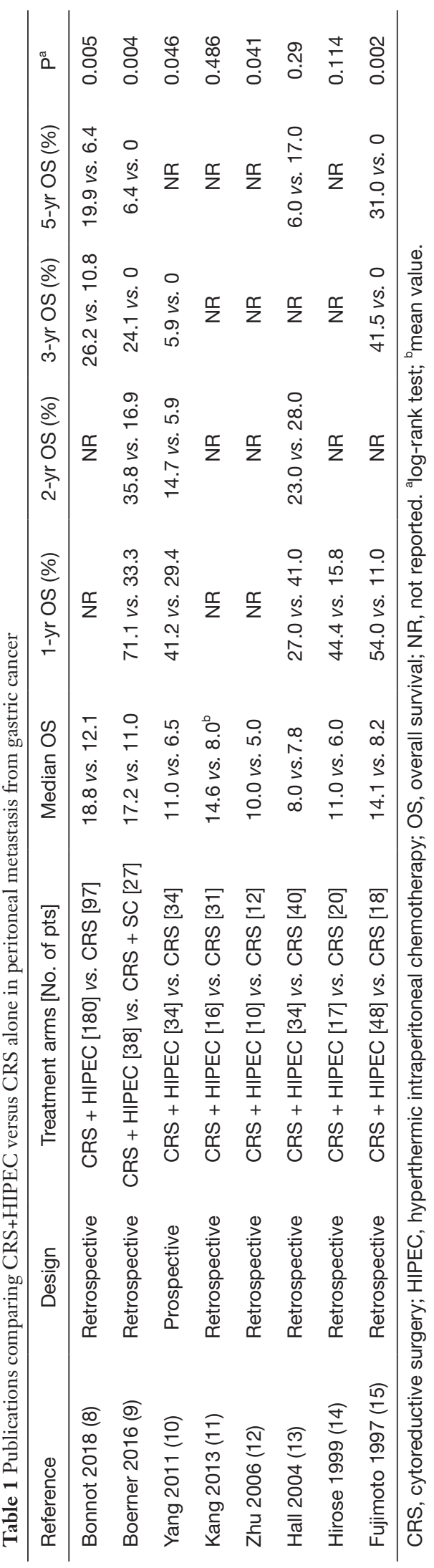

Thereafter, we tried lobaplatin + docetaxel combination in an open HIPEC technique. $50 \mathrm{mg} / \mathrm{m}^{2}$ of lobaplatin and $60 \mathrm{mg} / \mathrm{m}^{2}$ of docetaxel was each were dissolved in $3 \mathrm{~L}$ of normal saline. HIPEC was performed at $43.0 \pm 0.5{ }^{\circ} \mathrm{C}$ for 60 minutes. This regiments also showed efficacy and safety in GCPM patients (26).

However, we then changed our HIPEC regimen due to some non-medical reasons, like health economic disadvantage of lobaplatin, and short supply of mitomycin C. Lobaplatin was replaced by Cisplatin for HIPEC.

Recently, we retrospectively analyzed 125 GCPM patients treated with CRS+HIPEC at our center and investigated the impact of different HIPEC regimens on OS. The median OS for HIPEC-drug combination of cisplatin + docetaxel, lobaplatin + docetaxel, and cisplatin + mitomycin C was 12.4 (95\% CI: 8.3-16.5) months, 10.9 (95\% CI: 7.5-14.3) months, and 8.4 (95\% CI: 3.6-13.1) months, respectively $(\mathrm{P}=0.073)$ (Figure 3A). Although, there was no significant difference among these three groups, the first two groups showed a better result in short term survival. In terms of temperature, the median OS was 10.7 (95\% CI: 9.1-12.3) months in $43{ }^{\circ} \mathrm{C}-\mathrm{HIPEC}$ group, significantly better than 9.3 (95\% CI: 6.0$12.5)$ months in $42^{\circ} \mathrm{C}$-HIPEC group $(\mathrm{P}=0.030)$ (Figure $\left.3 B\right)$. In terms of duration, the median OS was 10.9 (95\% CI: 7.3-14.5) months in 60-minute group, significantly better than 10.3 (95\% CI: 8.1-12.4) months in 90-minute group $(\mathrm{P}=0.026)$ (Figure 3C). However, these factors showed no independently significant effect on OS.

\section{Conclusion}

Theoretically, CRS+HIPEC is designed as an integrated therapeutic package for peritoneal surface malignancies for cytologically radical resection and possible cure, with CRS for macroscopic resection of tumor nodules and HIPEC for microscopic eradication of IFCCs and micrometastasis. The recent PRODIGE 7 trial demonstrated an impressive advantage of complete CRS, but failed to verify the efficacy of HIPEC. This trial brings us a realization that we did not pay enough attention to HIPEC. It was discouraging that, we had not enough evidence from these publications and our own experience to conclude a recommended HIPEC regimen for GCPM. There is urgent need for standardizing HIPEC protocols worldwide. Accordingly, more international collaborations focusing on pharmacology and HIPEC-related parameters to generate high level evidence are essential. 
Table 2 Hyperthermic intraperitoneal chemotherapy regimens in publications

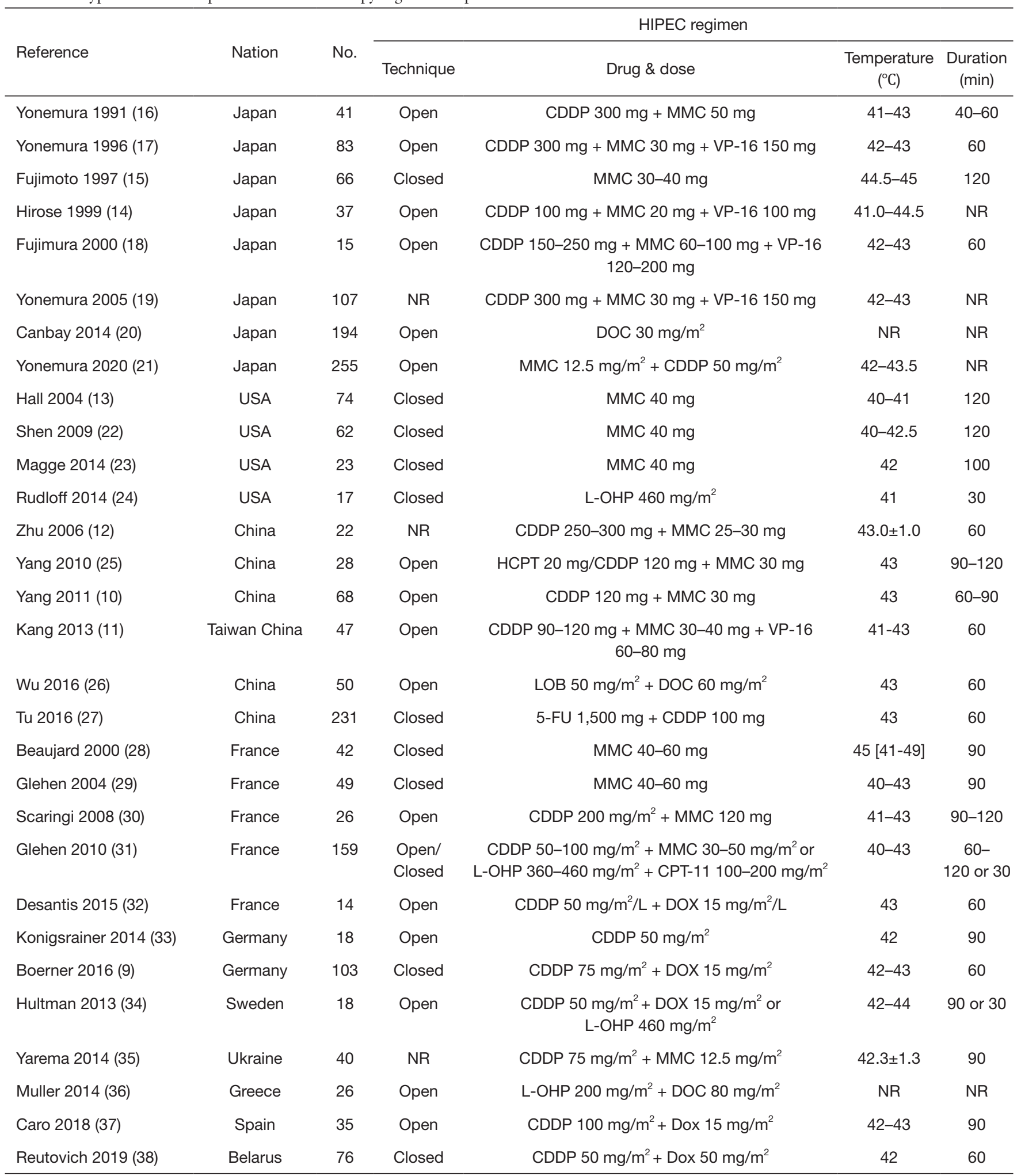

MMC, mitomycin C; CDDP, cisplatin; VP-16, etoposide; L-OHP, oxaliplatin; HCPT, hydroxycamptothecine; LOB, lobaplatin: DOC, docetaxel: 5-FU, 5-Fluorouracil; DOX, doxorubicin; CPT-11, irinotecan; NR, not reported. 

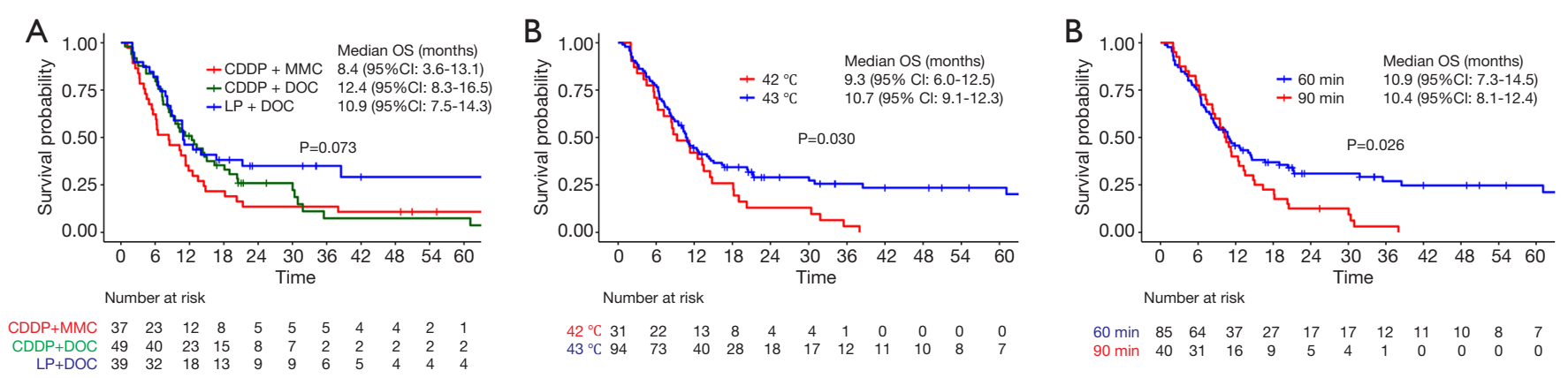

Figure 3 Kaplan-Meier curves and comparations of hyperthermic intraperitoneal chemotherapy (HIPEC) regimens: drugs (A), temperature (B) and duration (C). CDDP, cisplatin; MMC, mitomycin C; DOC, docetaxel; LP, lobaplatin.

\section{Acknowledgments}

Funding: This work was supported by Beijing Municipal Administration of Hospitals' Ascent Plan (DFL20180701).

\section{Footnote}

Provenance and Peer Review: This article was commissioned by the Guest Editors (Paul H. Sugarbaker and Kurt Van der Speeten) for the focused issue "Intraperitoneal Chemotherapy for Peritoneal Metastases: HIPEC, EPIC, NIPEC, PIPAC and More" published in Fournal of Gastrointestinal Oncology. This article has undergone external peer review.

Reporting Checklist: The authors have completed the Narrative review checklist. Available at http://dx. doi. org/10.21037/jgo-20-262

Conflicts of Interest: All authors have completed the ICMJE uniform disclosure form (available at http://dx. doi. org/10.21037/jgo-20-262). The focused issue was sponsored by the Peritoneal Surface Oncology Group International (PSOGI). The authors have no other conflicts of interest to declare.

Ethical Statement: The authors are accountable for all aspects of the work in ensuring that questions related to the accuracy or integrity of any part of the work are appropriately investigated and resolved.

Open Access Statement: This is an Open Access article distributed in accordance with the Creative Commons Attribution-NonCommercial-NoDerivs 4.0 International License (CC BY-NC-ND 4.0), which permits the non- commercial replication and distribution of the article with the strict proviso that no changes or edits are made and the original work is properly cited (including links to both the formal publication through the relevant DOI and the license). See: https://creativecommons.org/licenses/by-nc-nd/4.0/.

\section{References}

1. Ji ZH, Peng KW, Yu Y, et al. Current status and future prospects of clinical trials on CRS + HIPEC for gastric cancer peritoneal metastases. Int $\mathrm{J}$ Hyperthermia 2017;33:562-70.

2. Sugarbaker PH. Cytoreductive surgery and hyperthermic intraperitoneal chemotherapy in the management of gastrointestinal cancers with peritoneal metastases: Progress toward a new standard of care. Cancer Treat Rev 2016;48:42-9.

3. Yonemura Y, Canbay E, Li Y, et al. A comprehensive treatment for peritoneal metastases from gastric cancer with curative intent. Eur J Surg Oncol 2016;42:1123-31.

4. Chicago Consensus Working Group. The Chicago Consensus on peritoneal surface malignancies: Management of gastric metastases. Cancer 2020;126:2541-6.

5. Ji ZH, Peng KW, Li Y. Intraperitoneal free cancer cells in gastric cancer: pathology of peritoneal carcinomatosis and rationale for intraperitoneal chemotherapy/hyperthermic intraperitoneal chemotherapy in gastric cancer. Transl Gastroenterol Hepatol 2016;1:69.

6. Yonemura Y, Kawamura T, Bandou E, et al. The natural history of free cancer cells in the peritoneal cavity. Recent Results Cancer Res 2007;169:11-23.

7. Ji Z, Sun J, Wu H, et al. Assessment of hyperthermic intraperitoneal chemotherapy to eradicate intraperitoneal 
free cancer cells. Transl Oncol 2016;9:18-24.

8. Bonnot PE, Piessen G, Kepenekian V, et al. Cytoreductive surgery with or without hyperthermic intraperitoneal chemotherapy for gastric cancer with peritoneal metastases (CYTO-CHIP study): A propensity score analysis. J Clin Oncol 2019;37:2028-40.

9. Boerner T, Graichen A, Jeiter T, et al. CRS-HIPEC prolongs survival but is not curative for patients with peritoneal carcinomatosis of gastric cancer. Ann Surg Oncol 2016;23:3972-7.

10. Yang XJ, Huang CQ, Suo T, et al. Cytoreductive surgery and hyperthermic intraperitoneal chemotherapy improves survival of patients with peritoneal carcinomatosis from gastric cancer: final results of a phase III randomized clinical trial. Ann Surg Oncol 2011;18:1575-81.

11. Kang LY, Mok KT, Liu SI, et al. Intraoperative hyperthermic intraperitoneal chemotherapy as adjuvant chemotherapy for advanced gastric cancer patients with serosal invasion. J Chin Med Assoc 2013;76:425-31.

12. Zhu ZG, Tang R, Yan M, et al. Efficacy and safety of intraoperative peritoneal hyperthermic chemotherapy for advanced gastric cancer patients with serosal invasion. A long-term follow-up study. Dig Surg 2006;23:93-102.

13. Hall JJ, Loggie BW, Shen P, et al. Cytoreductive surgery with intraperitoneal hyperthermic chemotherapy for advanced gastric cancer. J Gastrointest Surg 2004;8:454-63.

14. Hirose K, Katayama K, Iida A, et al. Efficacy of continuous hyperthermic peritoneal perfusion for the prophylaxis and treatment of peritoneal metastasis of advanced gastric cancer: evaluation by multivariate regression analysis. Oncology 1999;57:106-14.

15. Fujimoto $S$, Takahashi M, Mutou T, et al. Improved mortality rate of gastric carcinoma patients with peritoneal carcinomatosis treated with intraperitoneal hyperthermic chemoperfusion combined with surgery. Cancer 1997;79:884-91.

16. Yonemura Y, Fujimura T, Fushida S, et al. Hyperthermochemotherapy combined with cytoreductive surgery for the treatment of gastric cancer with peritoneal dissemination. World J Surg 1991;15:530-5; discussion 535-6.

17. Yonemura Y, Fujimura T, Nishimura G, et al. Effects of intraoperative chemohyperthermia in patients with gastric cancer with peritoneal dissemination. Surgery 1996;119:437-44.

18. Fujimura T, Yonemura Y, Nakagawara H, et al. Subtotal peritonectomy with chemohyperthermic peritoneal perfusion for peritonitis carcinomatosa in gastrointestinal cancer. Oncol Rep 2000;7:809-14.

19. Yonemura Y, Kawamura T, Bandou E, et al. Treatment of peritoneal dissemination from gastric cancer by peritonectomy and chemohyperthermic peritoneal perfusion. Br J Surg 2005;92:370-5.

20. Canbay E, Mizumoto A, Ichinose M, et al. Outcome data of patients with peritoneal carcinomatosis from gastric origin treated by a strategy of bidirectional chemotherapy prior to cytoreductive surgery and hyperthermic intraperitoneal chemotherapy in a single specialized center in Japan. Ann Surg Oncol 2014;21:1147-52.

21. Yonemura Y, Prabhu A, Sako S, et al. Long term survival after cytoreductive surgery combined with perioperative chemotherapy in gastric cancer patients with peritoneal metastasis. Cancers (Basel) 2020;12:116.

22. Shen P, Stewart JHt, Levine EA. Cytoreductive surgery and intraperitoneal hyperthermic chemotherapy for peritoneal surface malignancy: non-colorectal indications. Curr Probl Cancer 2009;33:168-93.

23. Magge D, Zenati M, Mavanur A, et al. Aggressive locoregional surgical therapy for gastric peritoneal carcinomatosis. Ann Surg Oncol 2014;21:1448-55.

24. Rudloff U, Langan RC, Mullinax JE, et al. Impact of maximal cytoreductive surgery plus regional heated intraperitoneal chemotherapy (HIPEC) on outcome of patients with peritoneal carcinomatosis of gastric origin: results of the GYMSSA trial. J Surg Oncol 2014;110:275-84.

25. Yang XJ, Li Y, Yonemura Y. Cytoreductive surgery plus hyperthermic intraperitoneal chemotherapy to treat gastric cancer with ascites and/or peritoneal carcinomatosis: Results from a Chinese center. J Surg Oncol 2010;101:457-64.

26. Wu HT, Peng KW, Ji ZH, et al. Cytoreductive surgery plus hyperthermic intraperitoneal chemotherapy with lobaplatin and docetaxel to treat synchronous peritoneal carcinomatosis from gastric cancer: Results from a Chinese center. Eur J Surg Oncol 2016;42:1024-34.

27. Tu Y, Tian Y, Fang Z, et al. Cytoreductive surgery combined with hyperthermic intraperitoneal chemoperfusion for the treatment of gastric cancer: A single-centre retrospective study. Int J Hyperthermia 2016;32:587-94.

28. Beaujard AC, Glehen O, Caillot JL, et al. Intraperitoneal chemohyperthermia with mitomycin $\mathrm{C}$ for digestive tract cancer patients with peritoneal carcinomatosis. Cancer 2000;88:2512-9.

29. Glehen O, Schreiber V, Cotte E, et al. Cytoreductive 
surgery and intraperitoneal chemohyperthermia for peritoneal carcinomatosis arising from gastric cancer. Arch Surg 2004;139:20-6.

30. Scaringi S, Kianmanesh R, Sabate JM, et al. Advanced gastric cancer with or without peritoneal carcinomatosis treated with hyperthermic intraperitoneal chemotherapy: a single western center experience. Eur J Surg Oncol 2008;34:1246-52.

31. Glehen O, Gilly FN, Arvieux C, et al. Peritoneal carcinomatosis from gastric cancer: a multi-institutional study of 159 patients treated by cytoreductive surgery combined with perioperative intraperitoneal chemotherapy. Ann Surg Oncol 2010;17:2370-7.

32. Desantis M, Bernard JL, Casanova V, et al. Morbidity, mortality, and oncological outcomes of 401 consecutive cytoreductive procedures with hyperthermic intraperitoneal chemotherapy (HIPEC). Langenbecks Arch Surg 2015;400:37-48.

33. Königsrainer I, Horvath P, Struller F, et al. Initial clinical experience with cytoreductive surgery and hyperthermic intraperitoneal chemotherapy in signet-ring cell gastric cancer with peritoneal metastases. J Gastric Cancer 2014;14:117-22.

34. Hultman B, Lind P, Glimelius B, et al. Phase II study of patients with peritoneal carcinomatosis from gastric cancer treated with preoperative systemic chemotherapy followed by peritonectomy and intraperitoneal chemotherapy. Acta Oncol 2013;52:824-30.

Cite this article as: Ji ZH, Zhang Y, Li Y. Intra-operative hyperthermic intraperitoneal chemotherapy for prevention and treatment of peritoneal metastases from gastric cancer: a narrative review. J Gastrointest Oncol 2021;12(Suppl 1):S70-S78. doi: 10.21037/jgo-20-262
35. Yarema RR, Ohorchak MA, Zubarev GP, et al. Hyperthermic intraperitoneal chemoperfusion in combined treatment of locally advanced and disseminated gastric cancer: results of a single-centre retrospective study. Int J Hyperthermia 2014;30:159-65.

36. Müller H, Hotopp T, Tofeili A, et al. Systemic chemotherapy using FLOT - regimen combined with cytoreductive surgery plus HIPEC for treatment of peritoneal metastasized gastric cancer. Hepatogastroenterology 2014;61:703-6.

37. Rihuete Caro C, Manzanedo I, Pereira F, et al. Cytoreductive surgery combined with hyperthermic intraperitoneal chemotherapy (HIPEC) in patients with gastric cancer and peritoneal carcinomatosis. Eur J Surg Oncol 2018;44:1805-10.

38. Reutovich MY, Krasko OV, Sukonko OG. Hyperthermic intraperitoneal chemotherapy in serosa-invasive gastric cancer patients. Eur J Surg Oncol 2019;45:2405-11.

39. Rau B, Brandl A, Piso P, et al. Peritoneal metastasis in gastric cancer: results from the German database. Gastric Cancer 2020;23:11-22.

40. Manzanedo I, Pereira F, Rihuete Caro C, et al. Cytoreductive surgery and hyperthermic intraperitoneal chemotherapy (HIPEC) for gastric cancer with peritoneal carcinomatosis: multicenter study of Spanish Group of Peritoneal Oncologic Surgery (GECOP). Ann Surg Oncol 2019;26:2615-21. 